\title{
Synthesis of PEGylated Magnetic Nanoparticles With Different Core Sizes
}

\author{
Jesse Trekker ${ }^{1,2}$, Karolien Jans ${ }^{1}$, Hanne Damm ${ }^{1}$, Dokus Mertens ${ }^{3}$, Thomas Nuytten ${ }^{3}$, Johan Vanacken ${ }^{3}$, \\ Victor Moshchalkov ${ }^{3}$, Jan D’Haen ${ }^{4}$, Tim Stakenborg ${ }^{1}$, Willem Van Roy ${ }^{1}$, Uwe Himmelreich ${ }^{2}$, and \\ Liesbet Lagae ${ }^{1,3}$ \\ ${ }^{1}$ Imec, Leuven, B-3001, Belgium \\ ${ }^{2}$ Department of Imaging and Pathology, Biomedical MRI/Mosaic, KU Leuven, 3000, Leuven, Belgium \\ ${ }^{3}$ Department of Physics, Solid State Physics and Magnetism, KU Leuven, 3000, Leuven, Belgium \\ ${ }^{4}$ Institute for Material Research, Hasselt University, 3500, Hasselt, Belgium
}

\begin{abstract}
Tailoring the properties of superparamagnetic nanoparticles (MNPs) is essential for various nano-based biological applications. Having control over the properties of the MNPs permits a maximum flexibility. Starting from monodisperse iron oxide MNPs produced by thermal decomposition, we report on the optimization and characterization of a first and second seed mediated growth step by varying the surfactant amount and by optimizing the heating steps. We demonstrate the ability to gradually increase the size of crystalline MNPs from 6 over 9 to $12 \mathrm{~nm}$ with an improving monodispersity as demonstrated by Transmission Electron Microscopy, Dynamic Light Scattering and X-ray diffraction. The magnetic properties of the MNPs, studied by Vibrating Sample Magnetometry, were in concert with their size increase. We also show the functionalization of these particles with polyethylene glycolated silanes, to render the MNPs stable in water. Different characterization techniques, namely Transmission Electron Microscopy, Dynamic Light Scattering, Fourier-transform InfraRed, Thermo gravimetric analysis and X-ray Photoelectron Spectroscopy, confirmed the successful engraftment of the silanes on the MNP's surface. In conclusion, the proposed route of step-wise synthesis in combination with silane functionalization allows fine tuning the physical properties of iron oxide MNPs for applications in an aqueous environment.
\end{abstract}

Index Terms - Functionalization, magnetic nanoparticle, nanoparticle synthesis, seed mediated growth.

\section{INTRODUCTION}

$\mathbf{M}$ AGNETIC NANOPARTICLES (MNPs) have recently been the subject of intensive research within various fields such as material science and life science [1], [2]. Especially in life sciences, MNPs have successfully been used as contrast agents in Magnetic Resonance Imaging [3], as markers in biosensor technology [4], [5], as labels in immunomagnetic purification assays [6] and even as therapeutic agents in cancer therapy, so called magnetothermia [7]-[9]. Each of these applications predefines different properties for the MNPs. Commercially available MNPs are often not suited for a particular application and difficult to further tune as their coating or core are fixed. Creating the MNPs from the bottom up allows full control over the MNP's properties. A first step in this approach is to synthesize the magnetic cores of the MNPs. Several methods have been described for MNP synthesis of which co-precipitation is the most commonly used route [10]. Although this technique allows high yields and water-dispersibility and is easy to perform, the produced particles are broadly distributed and the size is difficult to control. Recently, more advanced techniques, producing high quality monodisperse MNPs have emerged of which the thermal decomposition method is widely applied [11]-[14]. In this solvothermal approach, a metal precursor is thermally decomposed in a high boiling solvent in the presence of stabilizing surfactants. This synthesis route leads to monodisperse MNPs because of a separation of the nucleation and growth phase, which is crucial in obtaining single sized MNPs [15]. The size of the MNPs can further be controlled by varying the reaction conditions, such as

Manuscript received July 09, 2012; revised September 10, 2012; accepted September 26, 2012. Date of current version December 19, 2012. Corresponding author: J. Trekker (e-mail: trekker@imec.be).

Digital Object Identifier 10.1109/TMAG.2012.2221687 the surfactant versus precursor ratio and the used solvent, or by seed-mediated growth. In the latter approach pre-synthesized seeds are added to the reaction mixture and are allowed to grow by the addition of an extra layer. This has been established for the synthesis of monodisperse $\mathrm{Au}, \mathrm{Fe}$ and $\mathrm{Fe}_{3} \mathrm{O}_{4}$ nanoparticles and even for nanoparticles of $\mathrm{Bi}, \mathrm{Sn}$, and In using $\mathrm{Au}$ particles as seeds [16]. More recent work examined the structural properties of seed mediated grown MNPs, indicating the presence of a magnetically frustrated layer, but did not focus on the synthesis parameters [17]. Different studies investigated the first seed growth and the influence of the precursor amount or the heating rate on the final size of the seed-grown MNPs [18], [19]. Even though this is important, the surfactant amount is even more crucial to obtain a reproducible synthesis.

An additional complication for these MNPs is their initial hydrophobic coating, which is not suited for water-based applications. This problem has been addressed by several authors using different coating materials, such as lipid bilayers [20], citric acid [21] or dextran [22] to enhance the MNPs' stability in polar solvents. Even though these coatings provide good stability at first sight, none of them rely on a covalent attachment to the iron oxide core. This means that in harsh conditions, such as in biological fluids or in cell vesicles, the coatings can be more easily desorbed from the surface, which hampers the long-term stability of the MNPs and can even enhance toxicity of the MNPs when used as cell label [20]. MNPs enwrapped by a coating attached by a covalent bond could overcome the drawbacks of the current applied coatings. It has been shown that silanes can successfully functionalize planar oxide surfaces [23] by covalent engraftment. Another advantage is that silanes are available with a variety of end-groups, allowing more advanced functionalities to be built in. Silane deposition on polydisperse MNPs synthesized in a watery environment (i.e., without initial hydrophobic coating) has already been reported [24], [25]. Few 
attempts, though, have been made on the silanization of hydrophobic MNPs [26]-[28].

Based on the above stated knowledge and shortcomings, the synthesis of high quality MNPs, produced by thermal decomposition, is presented in this manuscript. The optimization and characterization of a first $\left(\mathrm{SMG}^{1}\right)$ and second seed mediated growth $\left(\mathrm{SMG}^{2}\right)$ step is analyzed in detail. To prepare the MNPs for water-based applications, the hydrophobic coating was in one step replaced by a hydrophilic polyethylene glycolated silane coating.

\section{EXPERIMENTAL PROCEDURES}

\section{A. Reagents}

All chemicals were used as commercially received. Iron (III) acetylacetonate $(\geq 99.9 \%), 1,2$-hexadecanediol (technical grade, $90 \%$ ), oleic acid $(90 \%)$, benzyl ether $(90 \%)$, acetic acid $(\geq 99.5 \%)$, potassium bromide $(\geq 99 \%)$, and $n$-hexane $(>99.0 \%)$ were purchased from Sigma-Aldrich (Steinheim, Germany), whereas oleylamine $(>70 \%)$ and extra-dry toluene were obtained from Acros (Geel, Belgium). Ethanol, acetone, and methanol were purchased from Honeywell (Sleeze, Germany), chloroform from Merck (Darmstadt, Germany), triethoxy(methoxypolyethyleneoxy)silane, $5 \mathrm{~K}$ (mPEGSi) from Laysan Bio, Inc. (AL, USA) and uranyl acetate from Agar Scientific Ltd. (Stansted, England). Phosphate Buffered Saline (PBS) $(150 \mathrm{mM})$ was bought from Invitrogen (Gent, Belgium).

\section{B. Synthesis of Seeds}

The synthesis procedure has been adopted from the thermal decomposition method, which has been previously reported [11]. Briefly, $2 \mathrm{mmol}$ of iron (III) acetylacetonate, $10 \mathrm{mmol}$ of 1,2-hexadecanediol, $6 \mathrm{mmol}$ of oleic acid and $6 \mathrm{mmol}$ of oleylamine were added to $20 \mathrm{ml}$ benzyl ether in a three neck flask and placed in a reflux setup. The obtained black-brown mixture was heated to $100^{\circ} \mathrm{C}$ under a flow of nitrogen. Subsequently, the flask was sealed and the mixture was further heated to $200^{\circ} \mathrm{C}$ under a nitrogen blanket. After incubation for 2 hours at $200^{\circ} \mathrm{C}$, the mixture was further heated to reflux (nearly $300^{\circ} \mathrm{C}$ ). After 1 hour at reflux, the mixture was left to cool down to room temperature. Under ambient conditions the MNPs were washed three times with ethanol while retaining the MNPs in the flask using a rare earth magnet. Finally, the MNPs were suspended in $\mathrm{n}$-hexane, centrifuged for $30 \mathrm{~min}$ to remove undispersed residues, and kept at $4^{\circ} \mathrm{C}$ until further use.

\section{Seed Mediated Growth}

The seed mediated growth was performed in similarity to the synthesis of the seeds. More specifically, $80 \mathrm{mg}$ of pre-synthesized seeds were added to a mixture of $2 \mathrm{mmol}$ of iron (III) acetylacetonate, $10 \mathrm{mmol}$ of 1,2-hexadecanediol and $20 \mathrm{ml}$ of benzyl ether. For the first growth step $\left(\mathrm{SMG}^{1}\right), 2 \mathrm{mmol}$ or 6 mmol of oleic acid and oleylamine surfactant were added to the mixture in a 3 neck flask and placed in a reflux setup. The mixture was heated to $125^{\circ} \mathrm{C}$ while kept under a nitrogen atmosphere. At this temperature, the n-hexane was allowed to evaporate for 30 minutes. Next, the set-up was sealed under a nitrogen blanket, the mixture was heated to $200^{\circ} \mathrm{C}$ and, after 1 hour, the temperature was raised further to reflux. After 30 minutes at reflux, the mixture was allowed to cool down to room temperature. Washing and purifying was similar to the seed synthesis method. In the second growth step $\left(\mathrm{SMG}^{2}\right)$, the same amounts of MNPs $(80 \mathrm{mg})$ and precursors were used as in the first growth step, and also $2 \mathrm{mmol}$ or $6 \mathrm{mmol}$ of oleic acid and oleylamine was used. After mixing all the precursors and adding them to a three neck flask, the mixture was heated to $125^{\circ} \mathrm{C}$ and the n-hexane was purged off using nitrogen. Subsequently, three different heating schemes were used. In a first heating scheme, the mixture was heated to $200^{\circ} \mathrm{C}$ for 1 hour and kept at reflux for 30 minutes. In a second heating scheme, the reflux step was prolonged to 2 hours maintaining the previous 1 hour step at $200^{\circ} \mathrm{C}$. In a third scheme, the $200^{\circ} \mathrm{C}$ step was removed and the mixture was immediately heated to reflux for 2 hours. Washing and purifying the MNPs was similar as above.

\section{Silane Functionalization}

For surface functionalization, $6 \mathrm{mg}$ of dried MNPs and 150 $\mathrm{mg}$ of mPEGSi were added to $10 \mathrm{ml}$ extra-dry toluene in a glass centrifuge tube. This mixture was sonicated (Branson 2510, Branson Ultrasonics, CT, USA) until all the mPEGSi was dissolved. Next, $3 \mu 1$ of acetic acid was added to catalyze the silanization reaction. After 1 hour of vigorous shaking, 30 $\mathrm{ml}$ of $\mathrm{n}$-hexane was added to precipitate the MNPs. After a brief centrifugation step (1 $\mathrm{min}, 5000 \mathrm{rpm})$, the supernatant was removed by decantation and the MNPs were resuspended in $3 \mathrm{ml}$ chloroform. This washing procedure was repeated with $3 \mathrm{ml}$ acetone and $3 \mathrm{ml}$ methanol, sequentially. The methanol was purged off using a flow of nitrogen, and finally, the MNPs were resuspended in $1 \mathrm{ml}$ of DI water.

\section{E. Magnetic Nanoparticle Characterization}

Transmission electron microscopy (TEM): For TEM analysis, the MNPs were dropped and dried onto a carbon-coated copper grid. TEM images were captured using a $300 \mathrm{kV} \mathrm{CM} 30$ instrument (Philips, Eindhoven, The Netherlands) equipped with a field emission gun electron source. A statistical analysis was carried out on several TEM images. For each sample, at least 100 MNPs were counted. For visualization of the organic coating surrounding the MNPs, the MNPs were dropped and dried on Alcian blue pretreated, pioloform and carbon coated copper grids followed by a negative staining with $2 \%$ uranylacetate [29].

Thermal gravimetric analysis (TGA): TGA was performed on a Q5000 IR (TA instruments, New Castle, DE, USA) under nitrogen atmosphere to study the organic coating of the MNPs. First, $100 \mu 1$ of MNPs were slowly heated to $80^{\circ} \mathrm{C}$ to remove all solvents. Next, the temperature was increased to $850^{\circ} \mathrm{C}$ with a heating rate of $20^{\circ} \mathrm{C} / \mathrm{min}$. Using the software provided by the manufacturer (Universal Analysis 2000, v 4.5 A), the weight difference was analyzed before and after burning off the organic coating.

Fourier-transform infrared (FTIR): $300 \mathrm{mg}$ of potassium bromide and approximately $1 \mathrm{mg}$ of MNPs before or after mPEGSi functionalization were mixed. This mixture was dried in a vacuum oven during 48 hours at $50^{\circ} \mathrm{C}$ and subsequently pressed to form a thin pellet, which was mounted in a IFS66/v 


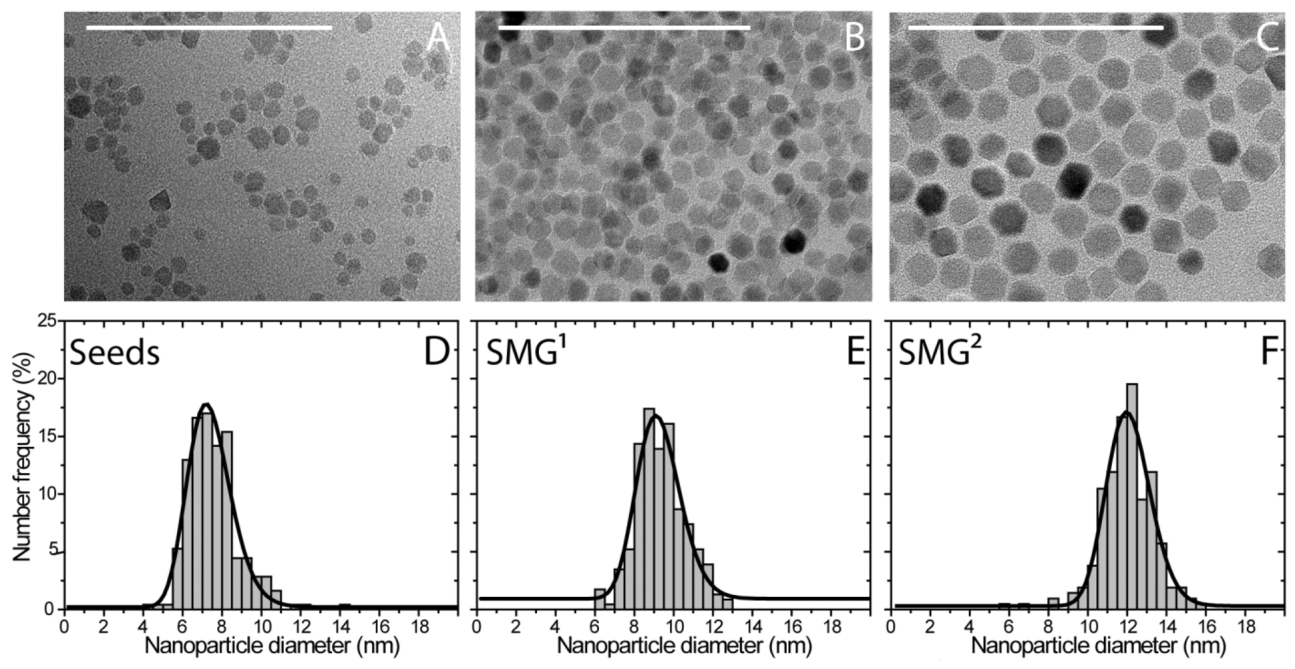

Fig. 1. (A)-(C) Bright field TEM images and (D)-(F) corresponding histograms of seed MNPs (A), (D), SMG ${ }^{1}$ (B), (E), and SMG ${ }^{2}$ nanoparticles (C), (F). The histograms, plotted as diameter versus number frequency, were fitted with a lognormal curve. Scale bar of the TEM images is $100 \mathrm{~nm}$.

FTIR device (Bruker Optik GmbH, Ettlingen, Germany). A pure $300 \mathrm{mg} \mathrm{KBr}$ pellet was used as background and was subtracted from the FTIR spectra of the MNP samples. Data were collected using 2048 scans between 4000 and $500 \mathrm{~cm}^{-1}$ at a resolution of $1 \mathrm{~cm}^{-1}$. The organic composition of the MNPs was investigated with OPUS software (v5.0).

$X$-ray diffraction $(X R D)$ : XRD diffractograms were obtained in a Bragg-Brentano configuration on an X'Pert Pro MRD instrument equipped with a $\mathrm{Cu} \mathrm{K} \alpha$ source and a PIXcel line detector (PANalytical B.V., Almelo, The Netherlands). MNPs were dried by evaporation on an amorphous silicon substrate to evaluate the crystal structure of the MNPs. Curve fittings to obtain FWHM and peak positions were performed in Igor Pro (v4.09A) and the crystallite size was calculated with X'Pert Data Viewer v1.2d using the Debye-Scherrer equation.

Dynamic Light Scattering (DLS) and zeta-potential: The intensity average size and zeta-potentials of the MNPs with and without functionalization were measured using a Zetasizer NanoZS instrument (Malvern, Leusden, The Netherlands) equipped with a red laser $(633 \mathrm{~nm})$ in backscatter mode $\left(173^{\circ}\right)$. The hydrodynamic diameter and the surface charge of the MNPs were investigated with the Zetasizer software v.6.01 provided by the manufacturer.

Vibrating Sample Magnetometer (VSM): Hysteresis, field-cooled (FC) and zero-field-cooled (ZFC) magnetization measurements were performed to study the magnetic properties of the MNPs using a VSM (Oxford Instruments, UK). The hysteresis curves were measured at $300 \mathrm{~K}$ and fitted with a Langevin function using Origin 8.1. For the ZFC measurement, the sample was cooled, starting above the blocking temperature, to $4 \mathrm{~K}$. Next, a field of 0.1 Tesla was applied and the ZFC curve was measured while heating the sample to $300 \mathrm{~K}$. After reaching $300 \mathrm{~K}$, the sample was cooled again to $4 \mathrm{~K}$ with an applied field of 0.1 Tesla. Subsequently, the FC curve was measured, while heating the sample to $300 \mathrm{~K}$.

$X$-ray Photoelectron Spectroscopy (XPS): XPS measurements were carried out in angle resolved mode using a Theta 300 system (ThermoInstruments, Waltham, MA, USA). The measurements were performed using a monochromatized $\mathrm{Al} \mathrm{K} \alpha$ $\mathrm{X}$-ray source $(1486.6 \mathrm{eV})$ and a spot size of 400 microns. A total of 16 spectra were recorded on MNPs dried on a gold substrate at an angle between 22 and $78^{\circ}$ measured from the surface normal to study the elements iron, oxygen, carbon and silicium. All spectra were fitted using Avantage software from ThermoInstruments.

Statistical Analysis: For every measurement a minimum of six analyses were performed.

\section{RESULTS}

\section{A. Magnetic Nanoparticle Synthesis}

1) Seed MNPs Produced by Thermal Decomposition: Using the thermal decomposition method as described [11], MNPs with a size of $6.9 \mathrm{~nm}$ (TEM) and a calculated size-dispersity of 0.19 [Fig. 1(A), (D)] were obtained. The hydrodynamic size measured by DLS was $14 \mathrm{~nm}$ (Table I). Using VSM, hysteresis curves were recorded, showing a typical s-shaped behavior of the magnetization versus applied field. No hysteresis could be measured, and the magnetization of the MNPs saturated at a field of approximately 0.5 Tesla [Fig. 2(A)]. From the FC and ZFC measurements the blocking temperature of the seeds was determined to be $50 \mathrm{~K}$ [Fig. 2(B)]. The crystal structure determination using XRD (Fig. 3) showed peaks at $2 \theta$ positions of approximately $30.2^{\circ}, 35.6^{\circ}, 43.2^{\circ}, 57.1^{\circ}$, and $62.7^{\circ}$. These peaks correspond to the (220), (311), (400), (511), and (440) planes of magnetite respectively.

2) First Seed Mediated Growth $\left(\mathrm{SMG}^{1}\right)$ : For the first seed mediated growth step, two amounts of surfactants were tested; $2 \mathrm{mmol}$ and $6 \mathrm{mmol}$ for both oleic acid and oleylamine. $2 \mathrm{mmol}$ rendered non-reproducible and often broadly distributed MNPs batches (data not shown). Increasing the amount of surfactant to $6 \mathrm{mmol}$ led to the formation of MNPs with a diameter of 9.9 $\mathrm{nm}$ as measured by TEM (Table I). In addition, the size-dispersity decreased to 0.13 [Fig. 1(B), (E)]. DLS measurements 

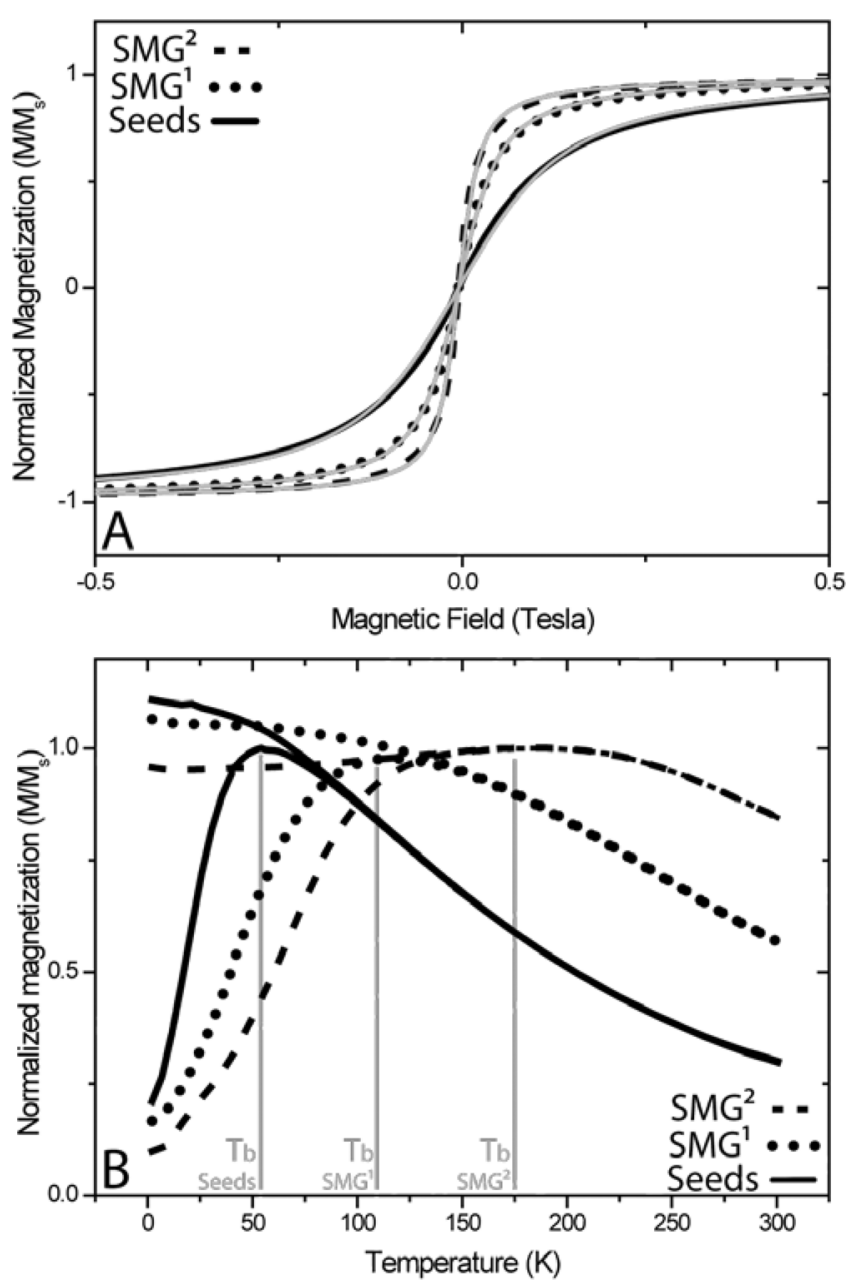

Fig. 2. (A) Normalized magnetic hysteresis curves of seed MNPs (full line), $\mathrm{SMG}^{1} \mathrm{MNPs}$ (dotted line), and $\mathrm{SMG}^{2} \mathrm{MNPs}$ (dashed line) measured at $300 \mathrm{~K}$ The grey curves are the corresponding Langevin curve fits. (B) Normalized FC and $\mathrm{ZFC}$ curves with the corresponding blocking temperature $\left(\mathrm{T}_{\mathrm{b}}\right)$, indicated by a grey line, for the different MNPs with an applied field of 0.1 Tesla.

also showed an increase in the MNP size from $14 \mathrm{~nm}$ for the seed MNPs to $18 \mathrm{~nm}$ for the $\mathrm{SMG}^{1}$ using $6 \mathrm{mmol}$ of surfactants (Table I). The magnetization of $\mathrm{SMG}^{1}$ particles saturated at lower fields compared to the seed MNPs, indicating a larger magnetic volume of the $\mathrm{SMG}^{1}$ MNPs [Fig. 2(A)]. The blocking temperature [Fig. 2(B)] increased to about $100 \mathrm{~K}$ for the $\mathrm{SMG}^{1}$, which is, similar to the hysteresis curve, related to a larger magnetic volume of the MNPs. The obtained XRD pattern for the $\mathrm{SMG}^{1}$ was comparable to the seed MNPs (Fig. 3), with the difference that more narrow diffraction peaks were obtained pointing towards larger crystal sizes of the grown MNPs.

3) Second Seed Mediated Growth $\left(\mathrm{SMG}^{2}\right)$ : If similar conditions to the optimized $\mathrm{SMG}^{1}$ synthesis were used for the second seed mediated growth step, MNPs were obtained, which were broadly distributed in size and with a clear bimodal size distribution (data not shown). Therefore, additional synthesis parameter modifications were tested to optimize this second seed mediated growth. TEM studies showed that $2 \mathrm{mmol}$ of oleic acid and oleylamine rendered broadly distributed MNP batches with no or little size increase (data not shown). Increasing the amount



Fig. 3. Measured XRD patterns of iron oxide nanocrystals of seed, $\mathrm{SMG}^{1}$, and $\mathrm{SMG}^{2}$ MNPs. The vertical lines in the diffractograms show the expected, theoretical peak positions for crystalline $\mathrm{Fe}_{3} \mathrm{O}_{4}$. The three patterns are offset vertically for clarity.

of surfactants to $6 \mathrm{mmol}$ produced bimodal distributions. By extending the time at reflux $\left(300^{\circ} \mathrm{C}\right)$ to 2 hours, the MNP size grew to $12.9 \mathrm{~nm}$ with a size-dispersity of 0.1 [Fig. 1(C), (F)]. Similar MNPs were obtained when omitting the intermediate heating step at $200^{\circ} \mathrm{C}$ and immediately heating the MNP mixture to $300^{\circ} \mathrm{C}$. DLS measurements showed a further increase of the MNPs size from $18 \mathrm{~nm}$ to $20 \mathrm{~nm}$ for $\mathrm{SMG}^{2}$ MNPs (Table I). The obtained hysteresis curves showed that the magnetization of $\mathrm{SMG}^{2}$ MNPs saturated at even lower fields as compared to $\mathrm{SMG}^{1}$ [Fig. 2(A)]. A further increase in the blocking temperature versus $\mathrm{SMG}^{1}$ was measured for the $\mathrm{SMG}^{2}$ in the order of 20-50 K, resulting in a blocking temperature of approximately 120 to $150 \mathrm{~K}$ [Fig. 2(B)]. The XRD diffractogram (Fig. 3) were again similar to the seeds and $\mathrm{SMG}^{1}$ particles with further increased peak intensities.

\section{B. Magnetic Nanoparticle Coating}

To render the MNPs hydrophilic, the hydrophobic MNPs were coated with a polyethylene glycolated silane. By studying the original hydrophobic coating of the MNPs and the new hydrophilic coating, the success of the functionalization was evaluated.

1) Hydrophobic MNPs: The amount of organic coating surrounding the hydrophobic MNPs was studied with different complementary techniques, i.e., TGA, FTIR and TEM. Using the weight percentage loss of the organic coating measured by TGA compared to the size determined by TEM, the amount of ligands $/ \mathrm{cm}^{2}$ can be obtained, resulting in similar values of $2.3-2.9 \times 10^{14}$ ligands $/ \mathrm{cm}^{2}$ for the seeds, $\mathrm{SMG}^{1}$, as well as for the $\mathrm{SMG}^{2}$ MNPs (Table I) [26]. Since TGA-data do not give direct information on the functional groups present in the coating of the MNPs, FTIR was used. A representative FTIR spectrum is shown in Fig. 4(C). For the hydrophobic MNPs a vibration band at $600 \mathrm{~cm}^{-1}$, which can be attributed to $\mathrm{Fe}-\mathrm{O}$ stretching vibrations [26], and two peaks at $1480 \mathrm{~cm}^{-1}$ and $1520 \mathrm{~cm}^{-1}$, specific for symmetric and asymmetric stretching vibrations of $\mathrm{COO}^{-}$could be observed [30]. Furthermore the 
TABLE I

PROPERTIES OF SYNTHESIZED MNPS

\begin{tabular}{|c|c|c|c|c|c|c|c|c|c|c|c|}
\hline \multirow{2}{*}{$\begin{array}{l}\text { Growth } \\
\text { step }\end{array}$} & \multicolumn{2}{|c|}{ TEM } & \multirow{2}{*}{$\begin{array}{l}\text { DLS } \\
\mathrm{D}_{\mathrm{DLS}} \\
(\mathrm{nm})\end{array}$} & \multirow{2}{*}{$\begin{array}{l}\mathbf{V S M}^{1} \\
\mathrm{D}_{\mathrm{VSM}} \\
(\mathrm{nm})\end{array}$} & \multirow{2}{*}{$\begin{array}{l}\mathbf{X R D}^{\mathbf{2}} \\
\mathrm{D}_{\mathrm{XRD}} \\
(\mathrm{nm})\end{array}$} & \multicolumn{6}{|c|}{$\mathrm{TGA}^{3}$} \\
\hline & $\begin{array}{l}\mathrm{D}_{\text {TEM }} \\
(\mathrm{nm})\end{array}$ & $\sigma$ & & & & $\begin{array}{c}\text { Weight loss } \\
(\%)\end{array}$ & $\begin{array}{c}\text { ligands } / \mathrm{cm}^{2} \\
\left(\times 10^{14}\right)\end{array}$ & ligands/MNP & $\begin{array}{c}\text { Weight loss } \\
(\%)\end{array}$ & $\begin{array}{c}\text { ligands/cm } \\
\left(\times 10^{14}\right)\end{array}$ & ligands/MNP \\
\hline Seeds & 6.9 & 0.19 & $14.2 \pm 2.4$ & $6.4-6.8( \pm 0.6)$ & 6.8 & $18.9 \pm 2.4$ & $2.3 \pm 0.3$ & $350 \pm 45$ & $35.1 \pm 6.7$ & $0.25 \pm 0.05$ & $37 \pm 7$ \\
\hline $\mathrm{SMG}^{1}$ & 9.9 & 0.13 & $18.2 \pm 2.9$ & $8.5-9.0( \pm 0.4)$ & 9.1 & $13.2 \pm 1.5$ & $2.3 \pm 0.3$ & $723 \pm 83$ & $24.0 \pm 4.2$ & $0.24 \pm 0.04$ & $74 \pm 13$ \\
\hline $\mathrm{SMG}^{2}$ & 12.9 & 0.1 & $20+5.3$ & $10.0-10.6(+0.4)$ & 12.1 & $12.6+2.0$ & $2.9+0.5$ & $1529+244$ & $21.3+1.5$ & $0.28+0.02$ & $146+10$ \\
\hline
\end{tabular}

MNP sizes and size distribution $\sigma$ determined by TEM, DLS, Langevin fitting of the VSM data $(n \geq 6) .{ }^{1}$ The range of Langevin diameters stated, were obtained by using the parameters for magnetite or maghemite. ${ }^{2}$ The XRD diameters were calculated using the Debye-Sherrer formula. ${ }^{3}$ Ligand coverage (left: oleic acid - right: mPEGSi) on the MNPs measured by TGA.
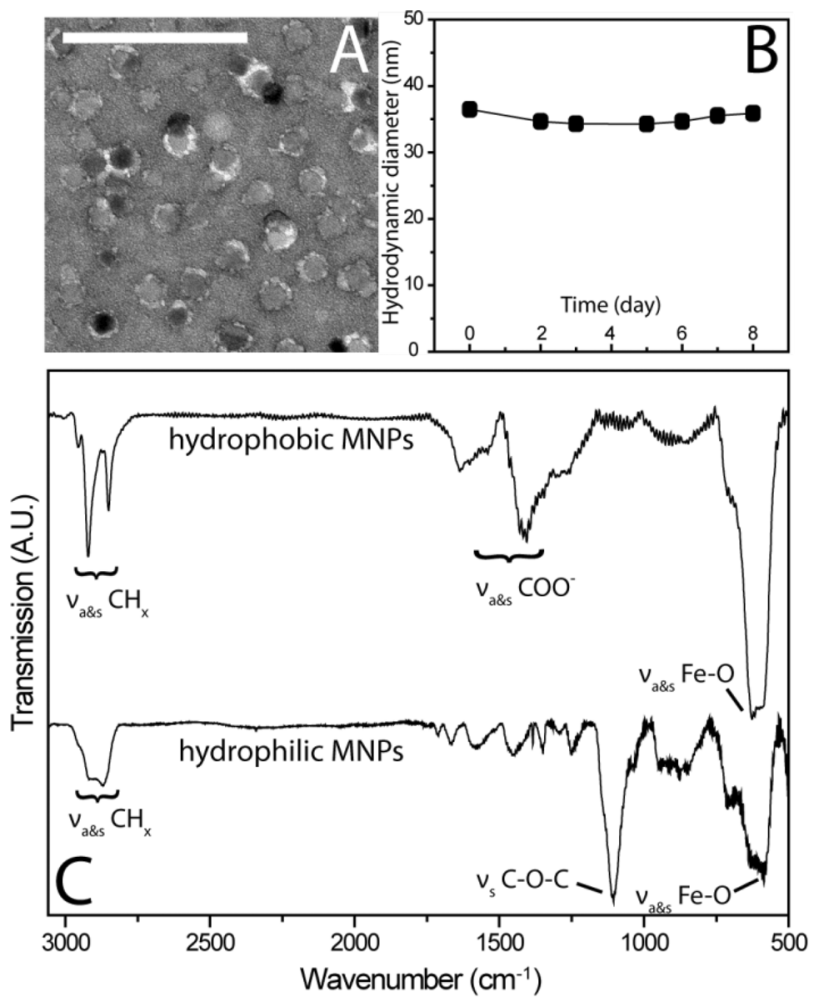

Fig. 4. (A) Background stained bright field TEM image of polyethylene glycolated silane functionalized MNPs. The figure clearly shows that the MNPs are enwrapped by a lighter, electron lucent layer. The scale bar is $100 \mathrm{~nm}$. (B) Evolution of the hydrodynamic diameter of the functionalized MNPs in PBS. (C) FTIR spectra of MNPs before and after silane functionalization. The spectra were offset vertically for clarity.

asymmetric and symmetric stretching vibrations of the alkane chains could be detected by several transmission bands at 2920 $\mathrm{cm}^{-1}$ and $2850 \mathrm{~cm}^{-1}$ respectively. More information on the chemical composition of the organic coating was obtained by XPS analysis of the elements $\mathrm{Fe}, \mathrm{C}, \mathrm{O}$, and $\mathrm{Si}$ (Table II). The Fe $2 p$ doublet with binding energies of 710.6 and $725.5 \mathrm{eV}$ could be seen in the $\mathrm{Fe}$ analysis, corresponding to the $2 \mathrm{p}_{1 / 2}$ and $2 \mathrm{p}_{3 / 2}$ binding energies, characteristic for iron oxide materials [26]. Furthermore, the deconvoluted high resolution XPS spectrum can provide more insight into the higher oxidation states for carbon with electronegative substituents. In the $\mathrm{C} 1 \mathrm{~s}$ subregion two peaks with binding energies 285 and $289.1 \mathrm{eV}$ were detected, typical for an alkane C-C bond and a C-O bond,
TABLE II

BINDING ENERGIES XPS

\begin{tabular}{ccc}
\hline \hline $\begin{array}{c}\text { XPS } \\
\text { subregion }\end{array}$ & $\begin{array}{c}\text { Hydrophobic MNPs } \\
\text { Binding energy (eV) }\end{array}$ & $\begin{array}{c}\text { Hydrophilic MNPs } \\
\text { Binding energy (eV) }\end{array}$ \\
\hline Fe $2 p$ & 710.6 & 710.3 \\
C 1s & 258.0 & 285.0 \\
C 1s & 289.1 & 286.4 \\
C 1s & - & 288.6 \\
O 1s & 530.0 & 529.9 \\
O 1s & 531.5 & 531.5 \\
O 1s & - & 532.8 \\
Si 2p & - & 101.8 \\
\hline \hline & Binding energies in the regions of Fe, C, O and Si atoms measured by \\
XPS for the synthesized MNPs and the functionalized MNPs.
\end{tabular}

respectively. In the $\mathrm{O} 1 \mathrm{~s}$ subregion spectrum a main peak of a $\mathrm{Fe}-\mathrm{O}$ bond was seen at $530 \mathrm{eV}$, and a small $\mathrm{COO}^{-}$bond peak could be found at $531.5 \mathrm{eV}$ [30]. No binding energies for $\mathrm{Si}$ could be detected (Table II).

2) Hydrophilic MNPs: After silane functionalization the hydrodynamic size of the MNPs, as measured by DLS, increased to approximately $40 \mathrm{~nm}$ and remained stable for at least 8 days in PBS [Fig. 4(B)]. The zeta-potential was slightly negative ( -3 $\mathrm{mV}$ ) at neutral $\mathrm{pH}$ (data not shown). To visualize the coating of the hydrophilic MNPs after functionalization a background stained bright field TEM image was taken [Fig. 4(A)]. Each MNP was enwrapped by an electron lucent coating layer. The amount of ligands $/ \mathrm{cm}^{2}$ was calculated based on the TGA- and TEM-data and was found to be $0.25-0.28 \times 10^{14}$ ligands $/ \mathrm{cm}^{2}$ for the seeds, $\mathrm{SMG}^{1}$ and $\mathrm{SMG}^{2}$ MNPs (Table I). TGA and TEM cannot elucidate the nature of the coating. Therefore, a FTIR spectrum was taken of the silane coated hydrophilic MNPs [Fig. 4(C)]. The hydrophilic MNPs spectrum showed a peak at $600 \mathrm{~cm}^{-1}$ for $\mathrm{Fe}-\mathrm{O}$ and an intense peak at $1100 \mathrm{~cm}^{-1}$, typical for symmetric stretching vibrations of C-O-C bonds [31]. Asymmetric and symmetric stretching vibrations of alkane chains were also measured at 2920 and $2850 \mathrm{~cm}^{-1}$. XPS measurements further elucidated the properties of the newly applied coating (Table II). No changes were seen in the Fe subregion, compared to the hydrophobic MNPs. The C 1s subregion, however, clearly showed two binding energies for the functionalized MNPs, one at $285.2 \mathrm{eV}$, indicative for a $\mathrm{C}-\mathrm{C}$ bond of the alkane chain and one at $286.4 \mathrm{eV}$, typical for $\mathrm{C}-\mathrm{O}$ bonds in polyethylene oxide chains [26]. In addition, a small peak at $288.6 \mathrm{eV}$ was detected, which indicated a $\mathrm{COO}^{-}$bond. The deconvoluted $\mathrm{O}$ 1s subregion had three distinguishable binding energies. The first at $529.9 \mathrm{eV}$ can be attributed to a $\mathrm{Fe}-\mathrm{O}$ bond. A second can be found at 531.5 $\mathrm{eV}$. As this binding energy is almost located at approximately 
$+2 \mathrm{eV}$ with respect to the main $\mathrm{Fe}-\mathrm{O}$ peak $(529.9 \mathrm{eV})$, it may be attributed to a Si-O-X bond or still a $\mathrm{COO}^{-}$bond [26], [32]. The binding energy at $532.8 \mathrm{eV}$ is characteristic for $\mathrm{C}-\mathrm{O}$ bonds. A small but detectable peak of oxidized silicon was seen in the $\mathrm{Si}$ XPS spectrum at $101.8 \mathrm{eV}$ [32].

\section{DISCUSSION}

\section{A. Magnetic Nanoparticle Synthesis}

The seed mediated growth process, as used here, is generally assumed to proceed in different stages [18]. In a first stage the nucleation of monomers takes place, forming MNP nuclei. Simultaneously with this primary nucleation, monomers attach to the surface of the pre-synthesized seeds leading to a growth of the MNPs. In addition, "defocusing" will occur following the classic Ostwald ripening process [18]. These effects of early nucleation and "defocusing" could be seen in our initial non-optimized growth steps as they rendered MNP batches with broad size distributions and no clear grown particle population (data not shown). In a second stage of the seed mediated growth process, monomers are exchanged between the smaller primary nucleated particles and the larger grown MNPs, i.e., the digestion ripening. In this stage the smaller MNPs gradually dissolve back into the solution, mainly because of a higher surface to volume ratio. The unstable surface atoms are released into solution and form a complex with the excess oleate ligand, making additional monomers available for the growth of larger MNPs [18]. The amount of surfactant thus plays a role in allowing a monomer exchange to occur between the small and the large MNPs. Therefore, the amount of surfactant was increased to $6 \mathrm{mmol}$ in the $\mathrm{SMG}^{1}$. A successful and monomodal size MNP batch was obtained. In addition a decrease of the polydispersity was achieved (Fig. 1). This effect of the surfactant, where iron-oleate contributes more to the growth of MNPs than to their nucleation, has also been mentioned in literature [16]. When applying similar reaction conditions to the $\mathrm{SMG}^{2}$, bimodal size distributions were obtained, although a clear grown population of MNPs was observed (data not shown). Therefore, the heating schemes of the $\mathrm{SMG}^{2}$ synthesis were extended to allow the smaller MNPs to be dissolved and the larger MNPs to grow. Two schemes were tested and both rendered monodisperse MNPs (Fig. 1).

1) Nanoparticle Size: To determine the quality and properties of the MNPs, different complementary techniques were used. TEM is the most straightforward method to determine the size and size distribution of the core MNPs (Fig. 1). Nonetheless, additional characterization methods are required as TEM cannot assess the internal crystalline or spin canting defects that can strongly affect the magnetic properties of the MNPs [17]. DLS confirmed the size of the MNPs in suspension (Table I). Larger nanoparticle sizes were obtained due to the fact that DLS measures the hydrodynamic diameter of the MNPs [26]. The XRD diffractograms of the different MNPs clearly indicated the crystalline nature of the MNPs (Fig. 3). The position of the peaks indicated the material to be magnetite or maghemite as the XRD patterns of both are similar. The narrowing of the diffraction peaks for the $\mathrm{SMG}^{1}$ and $\mathrm{SMG}^{2}$ MNPs indicated larger crystals for these MNPs. This can be more accurately quantified based upon the peak broadening and can be calculated using the Debye-Scherrer equation. An underestimation of the grain size of the MNPs can occur when neglecting other possible contributions to peak broadening such as the inherent system broadening of the XRD apparatus. The grain size of the seed particles correlated nicely with the size determined by TEM, giving only a minor difference of $0.1 \mathrm{~nm}$. The sizes of the magnetic core, calculated based upon the crystal grain for $\mathrm{SMG}^{1}$ and $\mathrm{SMG}^{2}$, were $0.8 \mathrm{~nm}$ smaller than determined by TEM. Overall the XRD results and TEM results are in concert with each other. The small decrease in size measured by XRD could indicate that the iron oxide layer deposited after seed mediated growth still consists of a small amorphous shell or a slightly different stoichiometry [33], [34]. This amorphous shell could have a negative impact on the magnetic properties of the MNPs, as the Fe atoms within this layer do not or only partially contribute to the magnetization of the MNP itself. In both cases however, $\mathrm{SMG}^{1}$ and $\mathrm{SMG}^{2}$, the XRD result indicated a size increase of the crystal core of the MNPs. As with the XRD data, the VSM data can also be used to determine the size of the magnetic core [13], [35], [36] (Fig. 2). Indeed, for a system in full superparamagnetic state the MNP magnetization $(\mathrm{M})$ versus an applied field $(\mathrm{H})$ can be described by the Langevin model:

$$
\begin{aligned}
\frac{M}{M_{s}} & =\operatorname{coth}\left(\frac{\mu_{0} \mu_{\mathrm{MNP}} H}{k_{B} T}\right)-\frac{k_{B} T}{\mu_{0} \mu_{\mathrm{MNP}} H} \\
& \equiv L\left(\frac{\mu_{0} \mu_{\mathrm{MNP}} H}{k_{B} T}\right)
\end{aligned}
$$

where $\mathrm{L}$ is the Langevin function, $\mu_{\mathrm{MNP}}$ is the magnetic moment of an individual $\mathrm{MNP}, \mathrm{M}_{\mathrm{s}}$ is the saturation magnetization of the system, $\mathrm{k}_{\mathrm{B}}$ is Boltzmann's constant, and $\mathrm{T}$ is the temperature in Kelvin. Using this equation to fit the normalized hysteresis curves, the total magnetic moment $\mu_{\mathrm{MNP}}$ of a single MNP can be calculated. Dividing $\mu_{\mathrm{MNP}}$ by the contribution of a single unit cell $\mu_{\text {cell }}$ (maghemite $80 / 3 \mu_{\mathrm{B}}$ or magnetite $32 \mu_{\mathrm{B}}$ ) gives the total number of unit cells within a MNP. Knowing the volume of one unit cell, the diameter of the MNPs can be calculated with the following equation:

$$
D_{\mathrm{VSM}}=\left(\frac{6 \mu_{\mathrm{MNP}} V_{\text {cell }}}{\pi \mu_{\text {cell }}}\right)^{1 / 3}
$$

where $\mathrm{D}_{\mathrm{VSM}}$ is the diameter determined by the hysteresis curve, $\mathrm{V}_{\text {cell }}$ is the volume of one unit cell, and $\mu_{\text {cell }}$ is the magnetic moment in one unit cell. Based upon the hysteresis curves [Fig. 2(A)] a magnetic diameter of $6.8 \mathrm{~nm}$ or $6.4 \mathrm{~nm}$ was found for the seed MNPs with respect to the parameters for maghemite or magnetite respectively. For the $\mathrm{SMG}^{1}$ MNPs $9.0 \mathrm{~nm}$ or $8.5 \mathrm{~nm}$ was calculated and $10.6 \mathrm{~nm}$ or $10.0 \mathrm{~nm}$ was obtained for the $\mathrm{SMG}^{2}$ MNPs. Using the parameters for maghemite delivered diameters closer to the results measured from TEM (Table I), indicating the presence of maghemite or a mixed phase with magnetite. A steady increase in magnetic core diameter was measured for the different MNPs. Similar to the XRD results the diameter of the magnetic core is smaller than the diameter measured with TEM (Table I). This result agrees with previous reports, where the smaller magnetic diameter versus structural diameter is attributed to a low magnetized shell caused by canted 
spins due to surface effects [33], [37] or due to the formation of a magnetically frustrated layer [17]. FC and ZFC measurements [Fig. 2(B)] indicated a steady increase of the magnetic blocking temperature in relationship to the volume of the MNPs, which is confirmed by literature [16]. Care must be taken with the FC and $\mathrm{ZFC}$ measurements of the $\mathrm{SMG}^{2} \mathrm{MNPs}$, as the shape of the field cooled measurement from $0 \mathrm{~K}$ to $100 \mathrm{~K}$ indicates some magnetic interactions between the MNPs. It was not possible to eliminate the interactions by lowering the sample concentration.

2) Monodispersity: In addition to the structural and magnetic size increase observed in TEM, DLS, XRD, and VSM, the size distribution $\sigma$ decreased from 0.19 for the seeds to 0.1 for the $\mathrm{SMG}^{2}$ (Table I). Interestingly, the absolute standard deviation on the size $( \pm 1.3 \mathrm{~nm})$, measured by TEM, was similar for the seeds, $\mathrm{SMG}^{1}$, and $\mathrm{SMG}^{2}$ MNPs. Under optimized synthesis conditions a uniform magnetic layer is deposited during the seed mediated growth. The size-dispersity is therefore determined by the size-dispersity of the seed MNPs.

\section{B. Magnetic Nanoparticle Coating}

Several applications utilizing MNPs, are water-based [38]. The thermal decomposition and seed mediated growth method rely on high boiling organic solvents. Consequently, the synthesized MNPs are only dispersible in hydrophobic solvents. A treatment of their surface is thus required to induce a transition from hydrophobic to hydrophilic. Two basic techniques can be applied to render hydrophobic MNPs hydrophilic, namely ligand addition and ligand exchange. The first method enwraps the MNPs including their original coating in a new shell, which introduces new surface properties. Because of the extra bulk and the loosely bound nature of these new ligands, a ligand exchange approach is preferred. In the latter approach, the original coating is replaced by a new coating. To induce such a transition, the new coating must be more tightly bound to the MNPs surface than the original one. As it is known that silanes can form a covalent bound with oxides [23], a polyethylene glycolated silane was chosen to functionalize the hydrophobic MNPs.

At first, the surface coating of the hydrophobic MNPs was investigated with FTIR, TGA and XPS. The FTIR data of the hydrophobic MNPs indicated the presence of iron oxide as the core material of the MNPs [Fig. 4(C)]. The carboxylate and alkane stretching vibrations showed the presence of oleic acid as stabilizing coating surrounding the MNPs, which also has been proven in previous reports [26], [30]. The XPS data (Table II) of the $\mathrm{Fe}$ subregion and $\mathrm{O} 1 \mathrm{~s}$ subregion confirmed the ferrite composition of the MNPs. The clear binding energies found in the $\mathrm{C}$ and $\mathrm{O}$ subregion further proved the presence of an organic layer on the nanoparticles. Similar to the FTIR data, the XPS results of the binding energies were typical for a carboxylate group in the $\mathrm{C} 1 \mathrm{~s}$ and $\mathrm{O} 1 \mathrm{~s}$ subregion, and the extra evidence for an alkane chain, support the findings that oleic acid coats the surface of the MNPs after synthesis. In addition, the TGA data confirmed the presence of such an organic layer around the MNPs. Knowing the fraction of oleic acid which is released versus the amount of MNPs introduced in the TGA system allows to calculate the number of oleic acid ligands enwrapping the MNPs. The amount of ligands per $\mathrm{cm}^{2}$ (surface coverage) was independent of the MNP size (Table I). The obtained value is in good agree- ment with previous reports, and in good agreement with literature data on self-assembled monolayers (SAMs) on 2D surfaces [26]. After silane deposition on the MNPs, the hydrodynamic diameter of the MNPs increased by about $30 \mathrm{~nm}$, which could indicate a small degree of aggregation. The MNPs retained this size for at least 8 days, while dispersed in PBS [Fig. 4(B)]. A bright field background stained TEM image was also taken of the hydrophilic MNPs [Fig. 4(A)], which showed that the MNPs were enwrapped by a shell of coating. The surface coverage of the silane, calculated based on the TGA-data, was lower at $0.24-0.28 \times 10^{14}$ ligands $/ \mathrm{cm}^{2}$ (Table I) compared to a previous report [26], but still dense enough to maintain a stable dispersion of the MNPs even in PBS [Fig. 4(B)]. More in depth FTIR analysis of the coating was performed [Fig. 4(C)]. The hydrophilic MNP FTIR data showed a similar peak for iron oxide compared to the hydrophobic MNPs, but had apart from that a clearly different spectrum, due to $\mathrm{C}-\mathrm{O}-\mathrm{C}$ stretching vibration bands. These last vibrations are abundant in the polyethylene groups of the silane. This was further confirmed by the XPS data, where a doublet appeared in the $\mathrm{C}$ 1s subregion (Table II). The binding energies could be attributed to the alkane chain and to the C-O bindings from the polyethylene groups. The additional small peak in the C1s subregion of the hydrophilic MNPs could, however, indicate that some oleic acid molecules are still present. The binding energy found in the Si subregion was typical for oxidized silicium, confirming the successful engraftment of the polyethylene glycolated silane on the MNPs [32].

\section{CONCLUSION}

In conclusion, the optimized synthesis of a first and second seed mediated growth process has been developed. By varying the heating scheme and the surfactant amount, monodisperse MNPs with sizes from 6.9 to $12.9 \mathrm{~nm}$ have been achieved. The careful characterization of the MNPs has shown to be crucial in optimizing the synthesis protocol to achieve high quality MNPs. In a last step, the successful engraftment of a polyethylene glycolated silane on the surface of the MNPs has been shown. Overall, this has resulted in an easy method to produce waterstable monodisperse MNPs with different sizes, which is essential for future biological and biomedical applications of those particles.

\section{ACKNOWLEDGMENT}

One author, J. Trekker, is the recipient of a research grant from the IWT-Vlaanderen. L. Lagae, V. Moshchalkov, J. D'Haen. and U. Himmelreich, are recipients of an IWT grant-sponsoring Project SBO80017. U. Himmelreich received financial support from the KU Leuven Program financing IMIR, from the European Commission for EC-FP7 network ENCITE (2008-201842), and from the "Vibrant" project (FP7-NMP-2008-228933). The authors thank Dr. J. Mast for the background stained TEM images and Dr. T. Conard and I. Hoflijk for the XPS measurements. The valuable help of T. Steylaerts with the MNP synthesis is also highly appreciated.

\section{REFERENCES}

[1] Y.-W. Jun, J.-W. Seo, and J. Cheon, "Nanoscaling laws of magnetic nanoparticles and their applicabilities in biomedical sciences," $A c$ counts Chem. Res., vol. 41, pp. 179-189, Feb. 2008. 
[2] N. A. Frey, S. Peng, K. Cheng, and S. Sun, "Magnetic nanoparticles: Synthesis, functionalization, and applications in bioimaging and magnetic energy storage," Chem. Soc. Rev., vol. 38, pp. 2532-2542, Sept. 2009.

[3] Y. Jun, J.-H. Lee, and J. Cheon, "Chemical design of nanoparticle probes for high-performance magnetic resonance imaging," Angew. Chem. Int. Edit., vol. 47, pp. 5122-5135, Jan. 2008.

[4] R. De Palma, G. Reekmans, W. Laureyn, G. Borghs, and G. Maes, "The optimization of magnetosandwich assays for the sensitive and specific detection of proteins in serum," Anal. Chem., vol. 79, pp. 7540-7548, Oct. 2007

[5] R. D. Palma, G. Reekmans, C. Liu, R. Wirix-Speetjens, W. Laureyn, O. Nilsson, and L. Lagae, "Magnetic bead sensing platform for the detection of proteins," Anal. Chem., vol. 79, pp. 8669-8677, 2007.

[6] H. L. Liu, C. H. Sonn, J. H. Wu, K.-M. Lee, and Y. K. Kim, "Synthesis of streptavidin-FITC-conjugated core-shell $\mathrm{Fe}_{3} \mathrm{O}_{4}-\mathrm{Au}$ nanocrystals and their application for the purification of CD4+ lymphocytes," Biomaterials, vol. 29, pp. 4003-4011, Oct. 2008.

[7] J.-P. Fortin, F. Gazeau, and C. Wilhelm, "Intracellular heating of living cells through Néel relaxation of magnetic nanoparticles," Eur. Biophys J., vol. 37, pp. 223-228, Feb. 2008.

[8] J. Jang, H. Nah, J.-H. Lee, S. H. Moon, M. G. Kim, and J. Cheon, "Critical enhancements of MRI contrast and hyperthermic effects by dopant-controlled magnetic nanoparticles," Angew. Chem. Int. Edit., vol. 48, pp. 1234-1238, Jan. 2009.

[9] R. Hergt, "Maghemite nanoparticles with very high AC-losses for application in RF-magnetic hyperthermia," J. Magn. Magn. Mater., vol. 270, pp. 345-357, Apr. 2004

[10] R. Massart, "Preparation of aqueous magnetic liquids in alkaline and acidic media," IEEE Trans. Magn., pp. 1980-1981, Aug. 1981.

[11] S. Sun, H. Zeng, D. B. Robinson, S. Raoux, P. M. Rice, S. X. Wang, and $\mathrm{G}$. $\mathrm{Li}$, "Monodisperse $\mathrm{MFe}_{2} \mathrm{O}_{4}(\mathrm{M}=\mathrm{Fe}, \mathrm{Co}, \mathrm{Mn})$ nanoparticles," J. Amer. Chem. Soc., vol. 126, pp. 273-279, Jan. 2004.

[12] J. Qin, S. Laurent, Y. S. Jo, A. Roch, M. Mikhaylova, Z. M. Bhujwalla, R. N. Muller, and M. Muhammed, "A high-performance magnetic resonance imaging T2 contrast agent," Adv. Mater., vol. 19, pp. 1874-1878, Jul. 2007.

[13] A. G. Roca, M. P. Morales, K. O’Grady, and C. J. Serna, "Structural and magnetic properties of uniform magnetite nanoparticles prepared by high temperature decomposition of organic precursors," Nanotechnology, vol. 17, pp. 2783-2788, June 2006.

[14] C.-M. Lee, H.-J. Jeong, S.-L. Kim, E.-M. Kim, D. W. Kim, S. T. Lim K. Y. Jang, Y. Y. Jeong, J.-W. Nah, and M.-H. Sohn, "SPION-loaded chitosan-linoleic acid nanoparticles to target hepatocytes," Int. J. Pharm., vol. 371, pp. 163-169, Apr. 2009.

[15] V. K. LaMer and R. H. Dinegar, "Theory, production and mechanism of formation of monodispersed hydrosols," J. Am. Chem. Soc., vol. 72, pp. 4847-4854, 1950 .

[16] J. Park, E. Lee, N.-M. Hwang, M. Kang, S. C. Kim, Y. Hwang, J.-G. Park, H.-J. Noh, J.-Y. Kim, J.-H. Park, and T. Hyeon, "One-nanometerscale size-controlled synthesis of monodisperse magnetic iron oxide nanoparticles," Angew. Chem. Int. Edit., vol. 44, pp. 2873-2877, May 2005.

[17] M. Levy, A. Quarta, A. Espinosa, A. Figuerola, C. Wilhelm, M. GarcíaHernández, A. Genovese, A. Falqui, D. Alloyeau, R. Buonsanti, P D. Cozzoli, M. A. García, F. Gazeau, and T. Pellegrino, "Correlating magneto-structural properties to hyperthermia performance of highly monodisperse iron oxide nanoparticles prepared by a seeded-growth route," Chem. Mater., vol. 23, pp. 4170-4180, 2011.

[18] J.-H. Huang, H. J. Parab, R.-S. Liu, T.-C. Lai, M. Hsiao, C.-H. Chen, H.-S. Sheu, J.-M. Chen, D.-P. Tsai, and Y.-K. Hwu, "Investigation of the growth mechanism of iron oxide nanoparticles via a seed-mediated method and its cytotoxicity studies," J. Phys. Chem. C, vol. 112, pp. 15684-15690, Oct. 2008.

[19] M. Lattuada and T. A. Hatton, "Functionalization of monodisperse magnetic nanoparticles," Langmuir, vol. 23, pp. 2158-2168, Mar 2007.
[20] S. J. H. Soenen, U. Himmelreich, N. Nuytten, T. R. Pisanic, A. Ferrari, and M. De Cuyper, "Intracellular nanoparticle coating stability determines nanoparticle diagnostics efficacy and cell functionality," Small, vol. 6, pp. 2136-2145, Oct. 2010

[21] A. Stroh, C. Zimmer, C. Gutzeit, M. Jakstadt, F. Marschinke, T. Jung, H Pilgrimm, and T. Grune, "Iron oxide particles for molecular magnetic resonance imaging cause transient oxidative stress in rat macrophages," Free Radical Bio. Med., vol. 36, pp. 976-984, Apr. 2004.

[22] C. Earhart, N. R. Jana, N. Erathodiyil, and J. Y. Ying, "Synthesis of carbohydrate-conjugated nanoparticles and quantum dots," Langmuir, vol. 24, pp. 6215-6219, June 2008.

[23] R. De Palma, W. Laureyn, F. Frederix, K. Bonroy, J.-J. Pireaux, G. Borghs, and G. Maes, "Formation of dense self-assembled monolayers of (n-decyl) trichlorosilanes on $\mathrm{Ta} / \mathrm{Ta}_{2} \mathrm{O}_{5}$," Langmuir, vol. 23, pp. 443-451, 2007.

[24] D. Forge, S. Laurent, Y. Gossuin, A. Roch, L. Vander Elst, and R. N. Muller, "An original route to stabilize and functionalize magnetite nanoparticles for theranosis applications," J. Magn. Magn. Mater., vol. 323, pp. 410-415, Mar. 2011.

[25] R. De Palma, J. Trekker, S. Peeters, M. J. Van Bael, K. Bonroy, R. Wirix-Speetjens, G. Reekmans, W. Laureyn, G. Borghs, and G. Maes, "Surface modification of gamma- $\mathrm{Fe}_{2} \mathrm{O}_{3} @ \mathrm{SiO}_{2}$ magnetic nanoparticles for the controlled interaction with biomolecules," J. Nanosci. Nanotechnol., vol. 7, pp. 4626-4641, Dec. 2007.

[26] R. De Palma, S. Peeters, M. J. Van Bael, H. Van den Rul, K. Bonroy, W. Laureyn, J. Mullens, G. Borghs, and G. Maes, "Silane ligand exchange to make hydrophobic superparamagnetic nanoparticles waterdispersible," Chem. Mater., vol. 19, pp. 1821-1831, Apr. 2007.

[27] Y. Sun, X. Ding, Z. Zheng, X. Cheng, X. Hu, and Y. Peng, "Surface initiated ATRP in the synthesis of iron oxide/polystyrene core/shell nanoparticles," Eur. Polym. J., vol. 43, pp. 762-772, Mar. 2007.

[28] C. Barrera, A. P. Herrera, and C. Rinaldi, "Colloidal dispersions of monodisperse magnetite nanoparticles modified with poly(ethylene glycol)," J. Colloid Interf. Sci., vol. 329, pp. 107-113, Jan. 2009.

[29] E. F. Van Doren, P.-J. R. H. De Temmerman, M. A. D. Francisco, and J. Mast, "Determination of the volume-specific surface area by using transmission electron tomography for characterization and definition of nanomaterials," J. Nanobiotechnol., vol. 9, p. 17, Jan. 2011.

[30] A. L. Willis, N. J. Turro, and S. O’Brien, "Spectroscopic characterization of the surface of iron oxide nanocrystals," Chem. Mater., vol. 17, pp. 5970-5975, Nov. 2005

[31] F. Hu, Q. Jia, Y. Li, and M. Gao, "Facile synthesis of ultrasmall PEGylated iron oxide nanoparticles for dual-contrast T1- and T2-weighted magnetic resonance imaging," Nanotechnology, vol. 22, p. 245604 , Jun. 2011

[32] M. R. Turner, E. Duguet, and C. Labrugère, "Characterization of silanemodified $\mathrm{ZrO}_{2}$ powder surfaces,” Surf. Interface Anal., vol. 25, pp. 917-923, Nov. 1997.

[33] A. Millan, A. Urtizberea, N. J. O. Silva, F. Palacio, V. S. Amaral, E. Snoeck, and V. Serin, "Surface effects in maghemite nanoparticles," J. Magn. Magn. Mater., vol. 312, pp. L5-L9, May 2007.

[34] F. Gazeau, E. Dubois, M. Hennion, R. Perzynski, and Y. Raikher, "Quasi-elastic neutron scattering on $\gamma-\mathrm{Fe}_{2} \mathrm{O}_{3}$ nanoparticles," Europhys. Lett., vol. 40, pp. 575-580, Dec. 1997.

[35] J. M. Vargas, L. M. Socolovsky, M. Knobel, and D. Zanchet, "Dipolar interaction and size effects in powder samples of colloidal iron oxide nanoparticles," Nanotechnology, vol. 16, pp. S285-S290, May 2005.

[36] R. Chantrell, J. Popplewell, and S. Charles, "Measurements of particle size distribution parameters in ferrofluids," IEEE Trans. Magn., vol. 14, no. 9, pp. 975-977, Sep. 1978.

[37] J.-F. Berret, O. Sandre, and A. Mauger, "Size distribution of superparamagnetic particles determined by magnetic sedimentation," Langmuir, vol. 23, pp. 2993-2999, Mar. 2007.

[38] A. K. Gupta and M. Gupta, "Synthesis and surface engineering of iron oxide nanoparticles for biomedical applications," Biomaterials, vol. 26, pp. 3995-4021, Jun. 2005. 